\title{
Diseño y evaluación de un taller de robótica basado en Estilos de Aprendizaje para la enseñanza de Fundamentos de Programación
}

\author{
Roberto Muñoz $^{1}$, René Nöel ${ }^{1}$, Marta Barría ${ }^{1}$, Thiago Barcelos ${ }^{2,3}$, Ismar Silveira ${ }^{3}$ \\ ${ }^{1}$ Escuela de Ingeniería Civil Informática - Universidad de Valparaíso \\ Av. Gran Bretaña, 1091, Playa Ancha, Valparaíso, Chile - CP 2360102 \\ ${ }^{2}$ Instituto Federal de Educação, Ciência e Tecnologia de São Paulo - IFSP \\ Av. Salgado Filho, 3501, Guarulhos, SP, Brasil - CEP 07115-000 \\ ${ }^{3}$ Universidade Presbiteriana Mackenzie \\ Rua da Consolação, 930 - São Paulo, SP, Brasil - CEP 01302-907 \\ roberto.munoz.s@uv.cl; rene.noel@uv.cl; marta.barria@uv.cl; \\ tsbarceloseifsp.edu.br; ismar@mackenzie.br
}

\begin{abstract}
Undergraduate disciplines related to programming fundamentals usually have high attrition rates. On the other hand, activities related to robotics may have a potential to motivate students as they bring a concrete, tangible application of programming skills. In this article we present the design and evaluation of a robotics workshop aimed at engineering students of a Chilean University that were simultaneously enrolled in introductory programming disciplines. An analysis of final grades indicated that students with higher attendance to workshop meetings had also higher grades in the programming discipline.
\end{abstract}

Resumen. Los cursos de pregrado relacionados con fundamentos de programación de computadores tienen, frecuentemente, altas tasas de reprobación. Por otro lado, el uso de robots tiene un alto potencial de permitir la aplicación más tangible y concreta de las habilidades de programación. En este artículo presentamos el diseño y evaluación de un taller de robótica, dirigido a estudiantes de Ingeniería de una Universidad chilena, que simultáneamente cursan la asignatura de Fundamentos de Programación. Los resultados de la primera ejecución del taller, indican que los alumnos con mayor asistencia al taller obtuvieron también mejores calificaciones finales en el curso de Fundamentos de Programación.

\section{Introducción}

En los últimos años, el nivel de retención de estudiantes de una universidad ha empezado a ser considerado un indicador para la medición de su calidad. De acuerdo las estadísticas nacionales de Chile, en cuanto a lo que respecta a la tasa de retención de la Universidad de Valparaíso (González et al. 2005), para primer año es de un 67,7\%, y para el segundo año es de un 49,9\%. En el caso de la Escuela de Ingeniería Civil Informática de la Universidad de Valparaíso (UV), la tasa de retención para primer año, cohorte 2013, fue de un 57,3\%, mientras que la tasa de titulación oportuna es tan sólo de un $5,9 \%$. 
El currículum estándar para cursos de pregrado de computación, de acuerdo a la ACM y la IEEE, define que el dominio de los fundamentos de programación de computadores es una habilidad esencial a ser desarrollada por los estudiantes (IEEE/ACM-CS, 2013). Los estudiantes de Ingeniería Civil Informática en la UV reciben su formación en programación a través de tres cursos: inicialmente, uno de Fundamentos de Programación (FP), que aborda tipos de datos, estructuras de control, y la definición y uso de algoritmos para la resolución de problemas. Posteriormente, y previa aprobación de FP, los alumnos cursan Programación I (P1), en el que se estudia el paradigma imperativo de programación, usando el lenguaje C. A Programación II, donde se estudia el paradigma de orientación a objetos, llega un número considerablemente menor de estudiantes que los que inician FP, esto debido a la alta tasa de deserción.

La introducción de robótica educacional es una de las estrategias posibles para incorporar nuevas tecnologías a los cursos de pregrado. Este enfoque ha sido recomendado por varios grupos de investigación como una herramienta para la enseñanza de ingeniería (Pittí et al., 2010; Sánchez et al., 2014). Esto se debe principalmente a que la robótica puede ser complementaria a numerosas áreas de conocimiento, tales como mecánica, electrónica, física y programación. De acuerdo a Monsalves (2011), el uso educacional de la robótica provee a los estudiantes un ambiente de aprendizaje empírico, con un notable potencial para el apoyo del aprendizaje activo El uso de kits de robótica prefabricados, tales como Lego Mindstorms NXT (Kelly, 2010), pueden además permitir a los estudiantes enfocarse en controlar el comportamiento del robot mediante la creación de programas computacionales. Como consecuencia, esta estrategia ayuda a los estudiantes de primer año a desarrollar sus habilidades de programación.

Por otro lado, la estrategia para incorporar robótica educacional a una asignatura de FP debe considerar la variedad de estilos de aprendizaje los estudiantes pueden tener. Mientras algunos estudiantes pueden preferir la exploración activa, otros pueden inclinarse por analizar y reflexionar antes de actuar, otros pueden preferir aprender escuchando o leyendo instrucciones, por nombrar algunos estilos (Felder \& Silverman, 1988).

Con base a las premisas previas, la UV ha desarrollado un taller de robótica, el que fue ofrecido a los estudiantes de primer y segundo semestre de la carrera de Ingeniería Civil Informática. Este artículo presenta el marco conceptual que sustenta el diseño del taller, y la descripción de la ejecución de sus actividades desde el año 2012. Se exponen también los resultados obtenidos, tanto en términos del análisis de sus calificaciones en las asignaturas de programación, como una evaluación del taller por parte de los alumnos, basada en un instrumento desarrollado para tal efecto.

\section{Trabajos relacionados}

Muchos grupos de investigación han estudiado el uso de la robótica como estrategia didáctica. Entre los beneficios frecuentemente identificados, se encuentran un aumento en la creatividad de los estudiantes, trabajo en equipo y autoaprendizaje. González y Jiménez (2010) presentan un experimento con kits de robótica de bajo costo para estimular en los estudiantes la exploración de relaciones entre la matemática, la física, la electrónica y la programación de computadores. Monsalves señala que un ambiente de 
aprendizaje adecuado para actividades didácticas con robots puede ayudar al aprendizaje de contenidos teóricos (Monsalves, 2011). El estudio de la robótica puede conducir a conexiones multidisciplinarias y actividades prácticas, tal como ha sido aplicado en carreras de ingeniería para apoyar la enseñanza de programación (Urrutia López et al., 2010; Ribeiro et al., 2011), sistemas de tiempo real (Malagelada, Toledo Morales, Valderrama Vallés, Sorribes Gomis, \& Pujol Capdevila, 2009), electrónica (Artal \& Artacho, 2011), entre otras materias. La mayoría de estos estudios describen experiencias a nivel de pregrado. Algunos de estos estudios indican que la robótica puede ayudar a los estudiantes a superar las dificultades iniciales del aprendizaje de la programación de computadores. Por ejemplo, en Fagin, Merkle \& Eggers (2001) se utiliza el lenguaje de programación ADA para programar robots Lego Mindstorms; los autores del estudio señalan que a los estudiantes se les hace más fácil entender el rol de los procedimientos y funciones en la descomposición de tareas, ya que ellos explícitamente piensan una función o un procedimiento como "una tarea que el robot debe llevar a cabo".

Por otro lado, incluso robots no tangibles pueden ser utilizados para lograr resultados positivos. (Becker (2001) describe el uso de un robot simulado que puede ser programado usando el lenguaje Java. Según el autor, el entorno de programación permite un primer contacto de los estudiantes con algunos conceptos de la programación orientada a objetos, como por ejemplo instanciación, llamadas a métodos y especialización de clases. Las estructuras de selectivas y reiterativas también pueden ser exploradas preliminarmente por los estudiantes.

En cuanto a los Estilos de Aprendizaje, de acuerdo a los diversos investigadores que han abordado el tema, existen diferentes modelos de representación. Por ejemplo, Keefe (1987) define que los estilos de aprendizaje como "rasgos cognitivos, afectivos y fisiológicos que sirven de indicadores relativamente estables de cómo las personas perciben, interactúan u responden a sus entornos de aprendizaje". Por otro lado, McCarthy y McCarhy (2005) señalan que las diferencias en de estilos de aprendizaje dependen de variados aspectos: quiénes somos, dónde estamos, cómo percibimos el mundo exterior y qué se requiere de nosotros. De acuerdo a esta definición, el estilo de aprendizaje de un individuo se describe en base a dos ejes: el eje acción/reflexión, y el eje de experiencia concreta/conceptos abstractos. Felder y Silverman (1988) proponen un modelo de estilos de aprendizaje para la ingeniería. De acuerdo a estos autores, los estilos de aprendizaje son definidos por las fortalezas y preferencias de los estudiantes, de acuerdo a la forma en que ellos procesan la información. Mientras algunos estudiantes se enfocan en la manipulación de datos y algoritmos, otros prefieren tratar con modelos matemáticos y teorías. Algunos estudiantes prestan más atención a las representaciones visuales como las ilustraciones, esquemas y diagramas; mientras otros prefieren obtener información en forma verbal o escrita. Algunos estudiantes adquieren mejores resultados al involucrarse en acciones activas y en la interacción con otros, mientras otros son más efectivos al trabajar en forma reflexiva e introspectiva. Este modelo fue adoptado en el presente estudio debido a la evidencia previa, que indicaba su eficiencia al ser aplicado en el diseño de actividades didácticas para estudiantes de ingeniería. 


\section{Diseño del taller}

Como se mencionó, FP y PI son el primer y segundo curso donde introducen conceptos de programación en los estudiantes de Ingeniería Civil Informática en la UV, respectivamente. Estas asignaturas tienen un alto grado de deserción, sobre un $65 \%$ en FP, y sobre un $45 \%$ en PI. Se aplicó una encuesta a los estudiantes para determinar posibles explicaciones a sus dificultades con estos cursos. Noventa y nueve estudiantes inscritos en las asignaturas de FP y PI respondieron la encuesta en mayo de 2012. En ese entonces, 51 de ellos habían aprobado FP y se encontraban cursando PI, mientras que los 48 restantes habían aprobado FP y PI. La encuesta contaba con preguntas acerca de la dificultad de las tareas ejecutadas por los estudiantes, de sus hábitos de estudio y de la calidad del material pedagógico. Todas las preguntas fueron respondidas en una escala de Likert de cinco valores.

Los resultados de la encuesta indican que los estudiantes prefieren aprender involucrándose en actividades prácticas por sobre actividades como asistir a clases o ejercicios en papel. Adicionalmente, los estudiantes tienen una preferencia casi igual hacia trabajar en solitario o en equipos. Estos resultados son similares a los obtenidos en otra encuesta, respondida por 44 estudiantes que ingresaron a la carrera de Ingeniería Civil Informática en el primer semestre de 2012. La encuesta se basó en el estándar de 44 preguntas propuesto por Felder y Silverman para identificar los estilos de aprendizaje. La mayoría de los estudiantes están balanceados entre ambas dimensiones, con una leve tendencia hacia el perfil Balanceado con Tendencia Activa (36\%), o Moderadamente Activo (21\%). Un $7 \%$ de los estudiantes fue caracterizado como Fuertemente Activo, mientras que un 2\% resultó ser Altamente reflexivo, un 14\% Balanceado con Tendencia Reflexiva y un 20\% Reflexivo Moderado. Estos datos parecen confirmar los resultados de la primera encuesta, indicando que los estudiantes de la carrera tienen una referencia similar entre el trabajo en solitario o en grupo, y tienen una fuerte inclinación a las actividades de aprendizaje prácticas. También, los resultados de la primera encuesta indican que los estudiantes se interesan más en los materiales didácticos de actividades de aprendizaje relacionadas con la dimensión Activa, tales como tutoriales y ejemplos.

Las otras dimensiones de aprendizaje identificadas como predominantes entre los estudiantes parecen ser consistentes con los resultados de la primera encuesta. Es posible inferir que la dimensión Sensitiva puede estar asociada a la preferencia de los estudiantes por las actividades prácticas. Por otro lado, la dimensión Secuencial puede estar asociada a la preferencia por materiales de enseñanza como tutoriales y ejemplos de programas. Aunque no podemos establecer una relación estadísticamente significativa entre ambas encuestas, esta puede ser de utilidad para proporcionar una idea sobre el perfil de los estudiantes con el fin de apoyar la definición de la estructura del taller.

\subsection{Estructura del Taller y materiales}

Con base a los resultados anteriores, se decidió definir las actividades del taller de manera de permitir la experimentación activa de los estudiantes en todo momento. Se consideró que la metodología de Aprendizaje Basado en Problemas (ABP) (Barrows, 1986) sería adecuada para este objetivo. En ABP, el proceso de aprendizaje se basa en la resolución de un problema no trivial propuesto inicialmente por el profesor. Con el fin de resolver el problema, los estudiantes deben organizarse en grupos pequeños y 
buscar autónomamente información que puede ayudar a resolver el problema (Barrows, 1986). El ABP también fue escogido debido a su potencial para ayudar a los estudiantes a desarrollar habilidades de trabajo en equipo y el auto-aprendizaje que son de gran valor en un contexto profesional.

Las actividades del taller se dividieron en cinco unidades, cada una compuesta por un conjunto de actividades. Los estudiantes debían completar todas las actividades de una unidad con el fin de avanzar a la siguiente unidad. Cada actividad fue diseñada para ser desarrollada en aproximadamente 90 minutos. Además, las actividades de cada unidad fueron diseñadas para involucrar un concepto fundamental de programación necesario para los estudiantes en las disciplinas de FP o P1. Los materiales didácticos para el taller fueron diseñados teniendo en cuenta las preferencias de aprendizaje de los estudiantes, identificadas previamente en la encuesta, y sus estilos de aprendizaje predominantes. Por lo tanto, los materiales fueron adecuados a los estilos de aprendizaje de los estudiantes de acuerdo a las dimensiones activas, secuenciales, visuales y de detección.

Aunque es posible identificar un estilo de aprendizaje predominante en base a los resultados de la encuesta, las diferencias individuales entre los estudiantes también deben ser consideradas en el diseño del taller. Como se supone que los estudiantes deben trabajar en equipo durante el taller, se definió una lista de los diferentes roles para cada estudiante, esto basado en una lista similar disponible en FIRST LEGO League (2012). Luego, los alumnos deberán intercambiar los papeles para que cada estudiante pueda desempeñar todas las funciones durante el taller.

Esta estrategia se basa en el constructivismo social (Bouzas, 2004; Luriia, Leontíev, Vygotskii, \& Benítez, 2009), una teoría que propone que el conocimiento se construye durante las interacciones sociales. Así, intercambiando sus roles, los estudiantes pueden ser capaces de aprender de las acciones y estrategias de sus compañeros. Cuando a un estudiante se le asigna inicialmente un rol (en el que supuestamente estará más cómodo), tendrá un mejor desempeño, por lo que todo el grupo podrá aprender de los puntos fuertes de cada uno.

\subsection{Validación del Taller}

El material y las actividades para el taller fueron validados en forma previa a la primera lección formal del taller. Para ello, se reclutó a 24 estudiantes voluntarios (12 estudiantes matriculados en FP y 12 alumnos matriculados en P1), que aceptaron participar en una versión más corta del taller. Este taller piloto se llevó a cabo durante dos meses. Debido a limitaciones de tiempo para el período de validación, después de ver una presentación más corta de la unidad, los estudiantes desarrollaron un único experimento por unidad. Cada presentación tomó unos 20 minutos, con otros 70 minutos asignados para el experimento. Los estudiantes recibieron los tutoriales de la unidad y las guías de actividades.

Al final de este taller piloto, los participantes respondieron a una encuesta sobre la calidad del material didáctico que recibieron. Se les preguntó acerca de la claridad de los materiales, qué tan bien estructurados están, y qué tan útiles fueron para el desarrollo de las actividades del taller. Cada dimensión de calidad fue evaluada por los estudiantes en una escala de 1 a 5 . Todas las dimensiones de calidad tuvieron un promedio entre 4,5 y 4,8 , lo que indica que el material de enseñanza fue bien aceptado por los estudiantes y, 
probablemente, podría ser utilizado en una ejecución del taller a mayor escala. La encuesta también planteó una pregunta abierta en la que los estudiantes podían expresar sus opiniones y sugerencias sobre el material didáctico. Los comentarios más frecuentes, sugirieron que el material debería incluir imágenes y vídeos más ilustrativos, así como más códigos de ejemplo. Estos comentarios fueron tomados en cuenta, ya que la estructura y el contenido del material didáctico fueron revisados y modificados en forma previa a la primera lección formal del taller.

\section{Resultados del taller}

La versión final del taller se ofreció a los estudiantes durante el segundo semestre del 2012. A todos los estudiantes de primer año matriculados en FP o P1 se les permitió participar en el taller, sin embargo, su participación no fue obligatoria. El taller contó con un proyecto final que se asignó a los estudiantes en función de su experiencia en programación. Se pidió a los estudiantes matriculados en FP que construyeran un Robot Patrullador, es decir, un robot que debe mantener el perímetro de una casa bajo vigilancia y detectar intrusos. A los estudiantes matriculados en $\mathrm{P} 1$ en tanto, se les pidió construir un Robot de Rescate, que debe pasar por un laberinto y encontrar un objeto negro puesto al azar dentro de él.

FP tuvo 25 estudiantes matriculados en el semestre, 15 de los cuales asistieron al menos a una de las sesiones de trabajo del taller. Los diez alumnos restantes que no participaron en ninguna de las sesiones del taller, también abandonaron el curso. En Programación 1 hubo 63 estudiantes matriculados, y 35 de ellos participaron en al menos una de las reuniones de trabajo. Los estudiantes de FP asistieron, en promedio, a un 33\% de las actividades del talleres, mientras que en los estudiantes P1 el promedio de asistencia fue de $71 \%$. Estos datos confirman que los estudiantes de P1 estaban más interesados en el taller; es necesario investigar si esto es un hecho aislado o si los estudiantes en el segundo semestre del curso pueden tener una mayor motivación para participar en un taller de este tipo.

\subsection{Análisis de las notas de los estudiantes}

Como se mencionó anteriormente, el taller fue una actividad opcional. Por este motivo, se optó por analizar las calificaciones obtenidas por los estudiantes en las asignaturas en comparación con su nivel de asistencia a las actividades del taller. Los resultados finales fueron agrupados en tres categorías para este análisis: alta asistencia $(\geq 75 \%$ de las actividades), media (de $45 \%$ a $74 \%$ de participación) o baja asistencia $(<45 \%)$. En la parte izquierda de la Figura 1 se muestra la distribución de las calificaciones de los participantes del taller matriculados en FP mientras en la parte derecha de la misma Figura se presenta la misma distribución para los estudiantes de P1.

En ambas disciplinas, el promedio la calificación final fue mayor para los grupos de estudiantes que tuvieron una mayor asistencia al taller (4,28 para los estudiantes de FP y 4,74 para los estudiantes de P1). El grupo de estudiantes de P1 con alta asistencia obtuvo altos promedios. También se observa que quienes poseen una alta asistencia al taller, ya se en FP o en P1, poseen una menor desviación estándar. Esto puede indicar que los estudiantes que participaron en la mayoría de las actividades del taller, a pesar de ser menos numerosos, son capaces de expresar y explicar los conceptos de programación durante sus evaluaciones. 

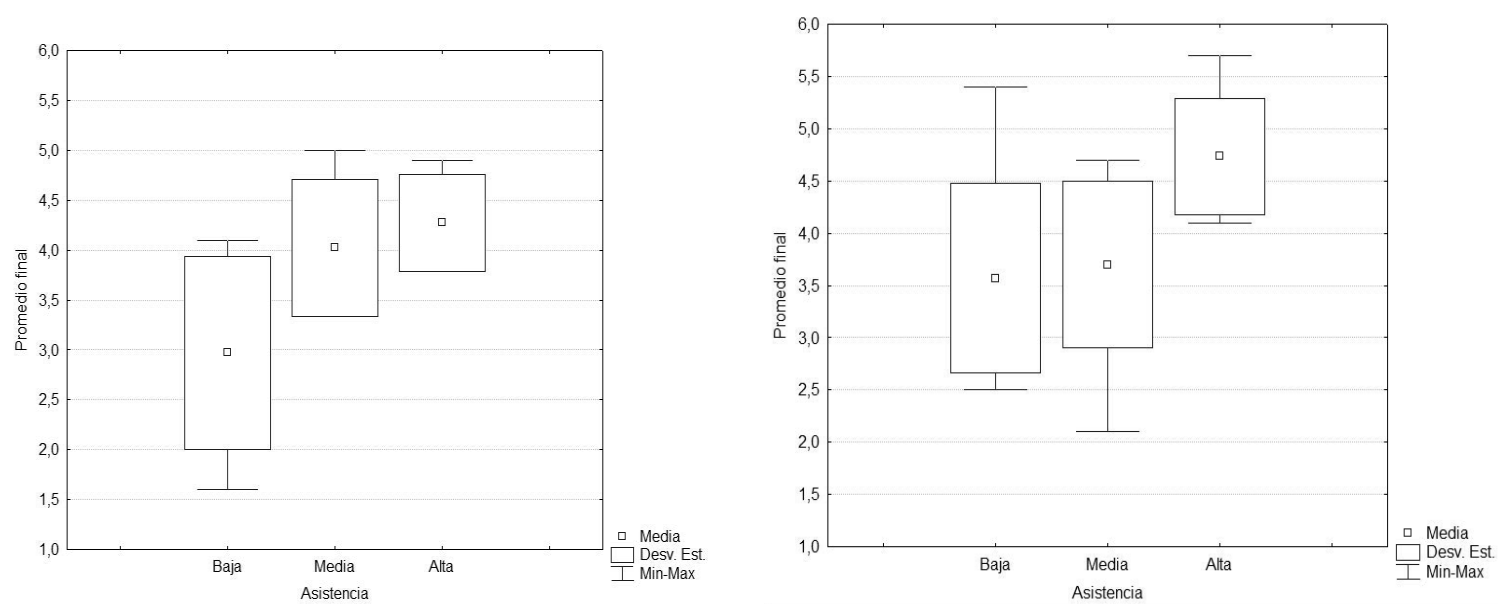

Figura 1. Distribución del promedio de los inscritos en F1 (izquierda) y P1 (derecha).

Con el fin de investigar la motivación de los estudiantes durante el taller, se llevó a cabo una encuesta para identificar ambos aspectos. La encuesta tenía once preguntas, definidas como frases positivas que a ser evaluadas por los estudiantes mediante una escala Likert de 5 valores. Los principales resultados sobre temas motivacionales están relacionados con las siguientes preguntas: (1) El taller de robótica incentiva el trabajo en equipo y el intercambio de conocimientos entre los estudiantes; (2) Estoy satisfecho con la forma en que estoy aprendiendo conceptos de programación (cátedra + clases en laboratorio + robótica); (3) Yo recomendaría asistir al taller de robótica a mis compañeros actuales y futuros; y (4) Aprender programación en el taller de robótica me ayuda a entender los temas tratados en la asignatura de programación.

Las preguntas 1 y 4 están relacionadas con cómo los estudiantes perciben los resultados de las actividades de aprendizaje; las preguntas 2 y 3 se centran en la satisfacción de los alumnos al participar de las actividades de aprendizaje. En el resto de esta sección se presentan y discuten los resultados obtenidos en estos cuatro puntos de la encuesta. Los resultados para las preguntas se presentan en la Figura 2.
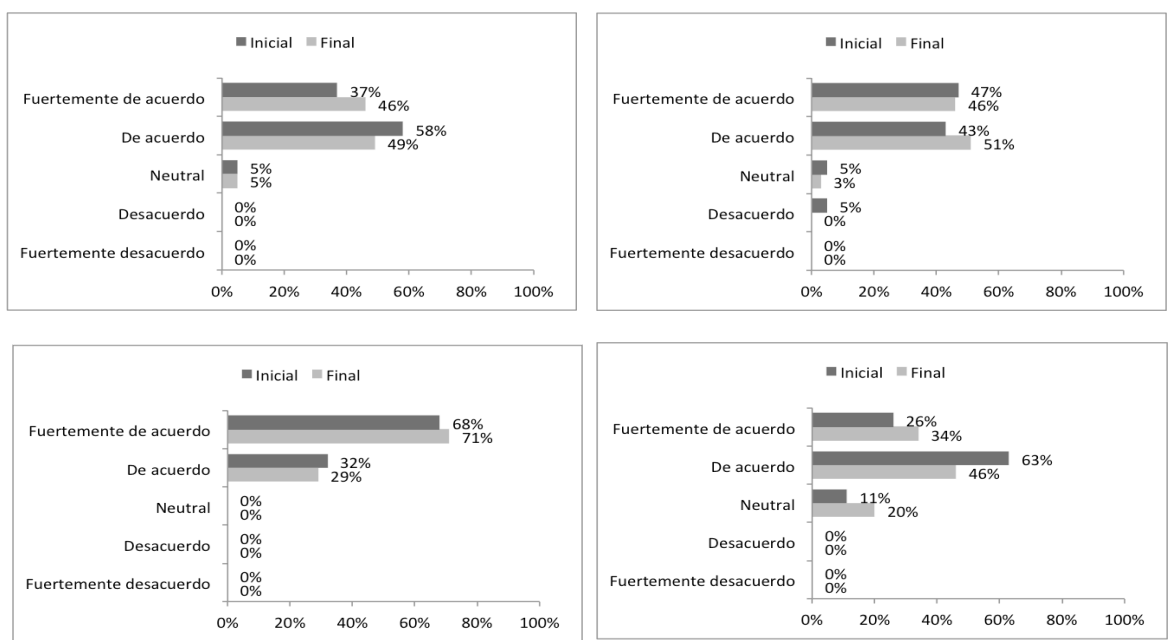

Figura 2. Respuestas a las preguntas 1 a 4 de la encuesta. 
Los estudiantes ya tenían una evaluación positiva del taller de acuerdo a la encuesta inicial, ya que no se registró ninguna evaluación neutra o negativa para esta afirmación. En la encuesta inicial sobre el perfil de los estudiantes se identificó una fuerte preferencia por las actividades de aprendizaje prácticas, lo que puede explicar este resultado. También, los estudiantes parecen estar más satisfechos después de haber finalizado las sesiones de taller, aumentando de esta forma la proporción de estudiantes que están totalmente de acuerdo con esta afirmación. Sin embargo, al parecer no es claro para los estudiantes cómo estas habilidades pueden ser transferidas a un entorno de programación "regular". Este hecho se puede observar en los resultados para la pregunta 4 (Figura 2, parte inferior derecha).

La proporción de estudiantes que están de acuerdo con la frase disminuyó en la segunda encuesta, y los estudiantes con una evaluación neutral de la frase se incrementó. Se necesitan estudios adicionales para identificar las posibles razones de este resultado. El trabajo previo en entornos alternativos para apoyar el aprendizaje de fundamentos de programación aportan alguna evidencia para explicar este resultado. Barak y Zadok (2009) concluyen que los estudiantes que participan en proyectos de robótica pueden no ser capaces de describir el proceso de resolución de problemas que utilizan, dado que la actividad estimula naturalmente el enfoque de ensayo y error. Por lo tanto, la obtención de una solución al problema puede ser más relevante para los estudiantes que entender por qué esa solución es la correcta. Una oportunidad de mejora sería incorporar en el diseño del taller instancias de discusión, guiados por el instructor, para ayudar a los estudiantes a consolidar los conceptos y reflexionar sobre los procedimientos que acaban de aplicarse.

La encuesta también tenía una pregunta opcional abierta, donde los estudiantes podrían dar sugerencias para mejorar el taller. La sugerencia más frecuente se relaciona con la duración del taller, ya que muchos estudiantes creen que las sesiones de trabajo serían más productivas si fueran más largas. Los estudiantes expresan también que los concursos de programación y las batallas de robots son altamente motivantes. Los estudiantes también sugirieron que sería muy bueno tener más kits de robótica disponibles. De los 10 kit que se tenían en ese momento, ya se han adquirido 30 kits para mejorar constantemente este aspecto del taller.

Las respuestas dadas por dos estudiantes pueden proporcionar evidencia adicional para dilucidar el cuestionamiento a la transferencia de habilidades discutido anteriormente: uno de ellos menciona que "Bricx tiene algunos elementos que no son iguales a C" ("Bricx Command Center," n.d.). Otro estudiante mencionó que durante el taller los estudiantes deben todavía "pensar en $C$ ". Esta evidencia indica que puede que no sea trivial para los estudiantes hacer frente a los detalles específicos de dos lenguajes de programación diferentes, al tiempo que aprenden las estructuras fundamentales de programación. Nuestro grupo de investigación está trabajando actualmente en un lenguaje de programación simplificado para Lego Mindstorms, para ayudar a los estudiantes a superar esta dificultad (Quiroz, Muñoz, \& Noël, 2012).

\section{Conclusiones y trabajos futuros}

Se desarrolló un taller de robótica para apoyar y complementar las dos asignaturas iniciales relacionadas con los conceptos de programación de computadoras (FP y PI) en la carrera de Ingeniería Civil Informática de la UV. Considerando que el diseño del 
taller se basa en la investigación previa del perfil y preferencias de aprendizaje de los estudiantes, así como sus estilos de aprendizaje predominantes, decidimos investigar el impacto del taller sobre el rendimiento y la motivación de los estudiantes. Los estudiantes con una mayor asistencia a las sesiones del taller también tuvieron mayores calificaciones finales en la asignatura de programación que se encontraban cursando en forma paralela. Una encuesta respondida por los estudiantes en la tercera y en las última sesión del taller indica que estos disfrutaron de las actividades del taller. Las actividades de resolución de problemas que involucran la interacción social y el intercambio de conocimientos también fueron apreciadas. Esto indica que las actividades de los talleres pueden motivar a los estudiantes y tener una influencia positiva en sus calificaciones. Sin embargo, se necesita investigar más en la influencia de aspectos motivacionales individuales, tales como la perseverancia y madurez, entre otros.

Creemos que las estrategias basadas en la resolución de problemas y la construcción de artefactos para apoyar el aprendizaje tienen un alto potencial y deben investigarse con mayor profundidad. Por esta razón, actualmente estamos desarrollando actividades de taller relacionadas con la construcción de otros artefactos, tales como juegos digitales. En futuros trabajos, tenemos la intención de entender mejor el papel de los estilos de aprendizaje y preferencias en la motivación de los estudiantes en la participación en actividades relacionadas con la construcción de artefactos.

\section{Referencias}

ACM-IEEE Software Engineering 2008. (2008). Curriculum Guidelines for Undergraduate Degree Programs in Software Engineering. IEEEComputer Society and Association for Computing Machinery.

Artal, J. S., \& Artacho, J. M. (2011). La Robótica como herramienta PBL en la enseñanza de la Electrónica en Ingeniería. In Actas I Congreso Internacional sobre Aprendizaje, Innovación y Competitividad. Madrid.

Barak, M., \& Zadok, Y. (2009). Robotics projects and learning concepts in science, technology and problem solving. International Journal of Technology and Design Education, 19(3), 289-307.

Barrows, H. S. (1986). A taxonomy of problem-based learning methods. Medical Education, 20(6), 481-486. http://doi.org/10.1111/j.1365-2923.1986.tb01386.x

Becker, B. W. (2001). Teaching CS1 with Karel the Robot in Java. SIGCSE Bull., 33(1), 50-54. http://doi.org/10.1145/366413.364536

Bouzas, P. (2004). El Constructivismo de Vigotsky: Pedagogía y Aprendizaje como Fenómeno Social. Longseller S.A. Retrieved from http://books.google.cl/books?id=j9cCAAAACAAJ

Bricx Command Center. (n.d.). Retrieved February 13, 2014, from http://bricxcc.sourceforge.net/

Escuela de Ingeniería Civil en Informática de la Universidad de Valparaíso. (2012, March). Inducción Alumnos. Retrieved from http://www.decomuv.documentos/induccionICI2012.html 
CBIE-LACLO 2015

Anais do XXI Workshop de Informática na Escola (WIE 2015)

Fagin, B. S., Merkle, L. D., \& Eggers, T. W. (2001). Teaching Computer Science with Robotics Using Ada/Mindstorms 2.0. Ada Lett., XXI(4), 73-78. http://doi.org/10.1145/507546.507592

Felder, R. M., \& Silverman, L. K. (1988). Learning and Teaching Styles in Engineering Education. Engineering Education, 78(7), 674-681.

FIRST LEGO League. (2012). Roles de equipo. Retrieved July 19, 2012, from http://www.firstlegoleague.cl

González, J., \& Jiménez, J. (2010). La robótica como herramienta para la educación en ciencias e ingeniería. Revista Iberoamericana de Informática Educativa, (10), 31-36.

González, L., Jorquera, D., \& González, S. (2005, April). Estudio sobre la repitencia y deserción en la educación superior chilena. Retrieved March 1, 2013, from

Keefe, J. W. (1987). Learning style: theory and practice. Reston: National Association of Secondary School Principals.

Kelly, J. F. (2010). Lego Mindstorms NXT-G Programming Guide. Apress. Retrieved from http://books.google.cl/books?id=-xbfc4ghj4YC

Luriia, A. R., Leontíev, A. N., Vygotskii, L. S., \& Benítez, M. E. (2009). Psicología y pedagogía. Madrid: Akal.

Malagelada, J., Toledo Morales, R., Valderrama Vallés, E., Sorribes Gomis, J., \& Pujol Capdevila, J. (2009). Un ABP basado en la robótica para las ingenierías informáticas. In Actas de las XV Jornadas de Enseñanza Universitaria de la Informática. Barcelona.

McCarthy, B., \& McCarthy, D. (2005). Teaching Around the 4MAT® Cycle: Designing Instruction for Diverse Learners with Diverse Learning Styles. Sage Publications.

Monsalves, S. (2011). Estudio sobre la utilidad de la robótica educativa desde la perspectiva del docente. Revista de Pedagogía, 32(90).

Pitti, K., Curto, B., \& Moreno, V. (2010). Experiencias construccionistas con robótica educativa en el Centro Internacional de Tecnologías Avanzadas. Teoría de La Educación: Educación Y Cultura En La Sociedad de La Información, 11(1).

Quiroz, P., Muñoz, R., \& Noël, R. (2012). Desarrollo de un Lenguaje de Programación y Entorno de Desarrollo que Facilite la Programación de Robots LEGO Mindstorms. In XVII Congreso Internacional de Informática Educativa 2012 (pp. 50-53). Santiago, Chile.

Sánchez, S., Rodríguez, O., \& Arribas, T. (2013). Utilización de LEGO NXT en la docencia universitaria. Retrieved July 19, 2014, from http://www.redusoi.org/

Urrutia López, H., Bustos Andreu, H., Villalobos Abarca, M., \& Jaramillo C, E. (2010). Aprendizaje de la programación mediante el uso de robot Lego por alumnos de computación de la Universidad de Tarapacá. In Actas del XXIV Congreso Chileno de Educación en Ingeniería. Valdivia, Chile. 